\title{
Update on Zika virus infection in pregnancy
}

\section{Atualização em Infecção Pelo vírus ZiKa NA GRAVIDEZ}

Ricardo Simões ${ }^{1,2}$, Renata Buzzini ${ }^{2}$, Wanderley Bernardo ${ }^{2}$, Florentino Cardoso $^{2}$, Antônio Salomão ${ }^{1}$, Giovanni Cerri ${ }^{2}$

1. Which factors may be related to the substantial increase in the number of cases of microcephaly in Brazil?

a. Increased active search for this congenital malformation.

b. Increase in the number of pregnant women traveling to northeastern Brazil.

c. Change in diagnostic criteria.

d. Alternatives a and c are correct.

2. What is the clinical and laboratory method indicated (sensitivity and specificity) to confirm Zika virus infection within a week of the onset of symptoms?

a. Immunoenzymatic assay (ELISA).

b. Plaque-reduction neutralization test (PRNT).

c. Reverse transcriptase technique.

d. Real-time polymerase chain reaction (RT-PCR).

e. Indirect immunofluorescence.

3. Regarding symptoms in pregnant women with suspected Zika virus infection, we know that:

a. Most are asymptomatic.

b. Patients can manifest fever, rash and fatigue.

c. Patients may present with purulent conjunctivitis, generalized joint pain, vomiting.

d. Alternatives $\mathrm{a}$ and $\mathrm{b}$ are correct.

4. What treatments are recommended for people infected with Zika virus?

a. Flu vaccine.

b. Analgesic agents, rest and antibiotics.

c. Hydration, rest and non-salicylic analgesic drugs.

d. Saline solution, anti-inflammatory drugs, good nutrition.

5. Regarding pregnant women diagnosed with Zika virus infection, we know that:

a. Care must be maintained according to conventional prenatal protocol if there are no symptoms, and wait until birth for other assessments. b. Fetal ultrasounds must be done in series every 3 to 4 weeks, and the newborn should receive complete physical examination with ophthalmologic assessment.

c. The patient should be referred for high-risk care at a prenatal service with specialized doctors, and the newborn should be evaluated by measuring the head circumference.

d. Evaluation should be done with frequent ultrasounds and, after birth, the newborn should have a head circumference of less than $34 \mathrm{~cm}$ to configure a need for more specific analyses.

Answers to Clinical scenario: Update ON CESAREAN DELIVERY AND SMALL NEWBORN FOR GESTATIONAL AGE [PUBLISHED IN RAMB 2016; 62(1)]

1. What is the most important single factor in neonatal mortality, in addition to being a significant determinant of postnatal mortality and infant morbidity? Birth weight. (Alternative D)

2. A retrospective study considered in this analysis suggested that SGA newborns exposed to labor have increased risk of early neonatal death compared with those not subject to labor. But in the same study, a lower risk of death was identified for what periods? For the late neonatal and postnatal periods in those born vaginally, regardless of gestational age. (Alternative $\mathbf{A}$ )

3. A retrospective study by Lee $\mathrm{HC}$ et al. showed inconsistent results; what was found?

SGA newborns with 31 weeks presented a decrease in late neonatal mortality at the time of cesarian section, while SGA newborns with a gestational age > 33 weeks, as well as those regarded as AGA (appropriate for gestational age), showed an increase in mortality in cases of cesarian section. (Alternative $\mathbf{B}$ ) 
4. According to Boers KE et al., was there any difference in neonatal mortality and morbidity, as assessed by Apgar score, among fetuses born vaginally or undergoing elective cesarean delivery?

There was no difference in neonatal mortality and morbidity. (Alternative $\mathbf{C}$ )
5. What can we say with regard to mode of delivery of small for gestational age fetuses in order to reduce neonatal and perinatal morbidity and mortality?

In the absence of other obstetric indications requiring cesarean section, there is no sufficient evidence to recommend planned cesarean section in the pregnancy of small for gestational age fetuses aiming to reduce neonatal and perinatal morbimortality. (Alternative $\mathbf{C}$ ) 